\title{
Theoretical Evaluation of Ultrasonic Velocities in Binary Liquid Mixtures of Anisaldehyde with Some Alcoxyethanols at Different Temperatures
}

\author{
Zareena Begum, P. B. Sandhya Sri, and C. Rambabu \\ Department of Chemistry, Acharya Nagarjuna University, Dr. M.R.Appa Rao Campus, Nuzvid, \\ Krishna District, Andhra Pradesh 521201, India \\ Correspondence should be addressed to Zareena Begum, begumzareena@gmail.com
}

Received 25 September 2012; Accepted 11 October 2012

Academic Editors: A. Tilocca and M. Tsige

Copyright ( $) 2012$ Zareena Begum et al. This is an open access article distributed under the Creative Commons Attribution License, which permits unrestricted use, distribution, and reproduction in any medium, provided the original work is properly cited.

\begin{abstract}
Ultrasonic velocities and densities of the binary liquid mixtures of anisaldehyde with alcoxyethanols like methoxyethanol (MOE), ethoxyethanol (EOE), and butoxyethanol (BOE) have been measured at temperatures $303.15 \mathrm{~K}, 308.15 \mathrm{~K}, 313.15 \mathrm{~K}$, and $318.15 \mathrm{~K}$ over the entire composition range of mole fractions. The theoretical values of ultrasonic velocity were evaluated using Nomoto's relation $\left(U_{\mathrm{NR}}\right)$, impedance relation $\left(U_{\mathrm{IR}}\right)$, ideal mixing relation $\left(U_{\mathrm{IMR}}\right)$, Jungie's relation $\left(U_{\mathrm{JR}}\right)$, and Rao's specific velocity relation $\left(U_{\mathrm{R}}\right)$. The molecular interaction parameter $(\chi)$ has been evaluated from the values of experimental and theoretical velocities. The variation of this interaction parameter with the composition mixture has been discussed in terms of molecular interactions.
\end{abstract}

\section{Introduction}

In recent years measurement of ultrasonic investigations found extensive applications in determining the Physicochemical behavior of liquid mixtures [1-5]. Several researchers [6-9] carried out ultrasonic investigations and correlated the experimental results of ultrasonic velocity with the theoretical relations of Nomoto [10, 11], van Deal and Vangeel ideal mix relations [12] impedance relation [13] Rao's Specific velocity [14] and Junjie [15] and interpreted the results in terms of molecular interactions. There has been an increasing interest in the study of intermolecular interactions in the last two decades and numbers of experimental techniques have been used to investigate the interactions between the binary liquid mixture components. ultrasonic study of liquid mixtures, due to its nondestructive nature, has been extensively carried out in different branches of science to measure the thermodynamic properties to predict the nature of molecular interaction between the molecules in a medium. The ultrasonic sound velocity and the thermodynamic parameters derived from it have been widely used to interpret the interactions between unlike molecules in the binary liquid mixtures.
Longeman and Correy [16] discussed sound velocity in a liquid as the sum of bond velocities.Rendall[17] has shown a close agreement between the experimental and theoretical values calculated from the adiabatic compressibility measurements. Auslander and Onitni [18], Samal and Misra [19], Aziz et al. [20, 21], and Younglove [22] showed that there is close relation between sound velocity and thermodynamic properties.

This investigation presents the evaluation of ultrasonic velocity using Nomoto's relation, ideal mixtures relation, impedance relation, Rao's specific velocity relation, and Junjie's relation for anisaldehyde and methoxyethanol, ethoxyethanol and butoxyethanol at temperatures of 303.15$318.15 \mathrm{~K}$ with intervals of $5 \mathrm{~K}$. An attempt has been made to study the molecular interactions from the deviation values in $U^{2} / U_{\text {imix }}^{2}$ from unity based on earlier studies $[23,24]$.

\section{Experimental}

Anisaldehyde (Merck India $>0.995$ purity) was distilled at low pressure and over freshly activated $0.3 \mathrm{~nm}$ molecular sieves [25]. The alcoxyethanols under study mentioned above 
(SD Fine Chemicals, India, with purity $>0.995$ ) were purified by methods described in the literature [26]. The purity of the chemicals was verified further by measuring the densities and ultrasonic velocities which are in good agreement with the literature values.

\section{Apparatus and Procedure}

The ultrasonic velocity of sound $(U)$ is measured using an ultrasonic interferometer (Mittal Enterprises, New Delhi model F05) operating at $2 \mathrm{MHz}$. The measured speeds of sound have a precision of $0.8 \mathrm{~m} \cdot \mathrm{sec}^{-1}$ and an uncertainty less than $\pm 0.1 \mathrm{~m} \cdot \mathrm{sec}^{-1}$. The temperature stability was maintained within $\pm 0.01 \mathrm{~K}$. By circulating water bath around the measuring cell through a pump.

The densities, $\rho$, of pure liquids and their mixtures are determined using a $10^{-5} \mathrm{~m}^{3}$ double-arm pycnometer, and the values from triplicate replication at each temperature are reproducible within $2 \times 10^{-1} \mathrm{~kg} \mathrm{~m}^{3}$ and the uncertainty in the measurement of density is found to be 2 parts in $10^{4}$ parts. The reproducibility in mole fractions was within \pm 0.0002 .

\section{Theory}

Comparison of theoretical values of ultrasonic velocities with those obtained experimentally in the present binary liquid mixtures is expected to reveal the nature of interaction between component molecules in the mixture. Such theoretical study is useful in finding the comprehensive theoretical model for the liquid mixtures.

\section{Nomoto's Relation $\left(U_{\mathrm{NR}}\right)$}

On assuming the additivity of molar sound velocity $(R)$ and no volume change on mixing, Nomoto established the following relation for the ultrasonic velocity of binary liquid mixtures:

$$
R=\frac{M}{\rho U^{1 / 3}}
$$

where $U$ and $\rho$ are determined experimentally, and $M$ is the mean molecular weight in a binary liquid mixture:

$$
M=\left(X_{1} M_{1}+X_{2} M_{2}\right) \text {, }
$$

where $M_{1}$ and $M_{2}$ are molecular weights of constituent components.

Simple manipulation yields the following relation:

$$
U_{\mathrm{NR}}=\left[\frac{\left(X_{1} R_{1}+X_{1} R_{2}\right)}{\left(X_{1} V_{1}+X_{2} V_{2}\right)}\right]^{3} .
$$

\section{The Impedance Relation $\left(U_{\mathrm{IR}}\right)$}

Impedance is the product of ultrasonic velocity $(U)$ and the density $(\rho)$ of a liquid mixture. Hence the impedance relation predicts the ultrasonic velocity of the given mixture by simply using the values of impedance $\left(Z_{i}\right)$ and the density $(\rho)$ values. Impedance relation is given as

$$
U_{\mathrm{IR}}=\frac{\sum X_{i} Z_{i}}{\sum X_{i} \rho_{i}}
$$

where $X_{i}$ mole fraction, $\rho_{i}$, is the density of the mixture and $Z_{i}$ is the acoustic impedance.

\section{The Jungie Equation $\left(U_{\mathrm{J}}\right)$}

The Junjie equation is given as

$$
U_{J}=\frac{\left(X_{1} M_{1} / \rho_{1}+X_{2} M_{2} / \rho_{2}\right)}{\left[\left\{X_{1} M_{1}+X_{2} M_{2}\right\}^{1 / 2}\left\{X_{1} M_{1} / \rho_{1} U_{1}^{2}+X_{2} M_{2} / \rho_{2} U_{2}^{2}\right\}^{1 / 2}\right]},
$$

where $M_{1}, M_{2}$ are molecular weights of constituent components. $\rho_{1}$ and $\rho_{2}$ are the densities of constituent components.

\section{The Rao's Specific Velocity Method Relation $\left(U_{R}\right)$}

Rao's specific velocity method [12] is given as

$$
U_{R}=\left(\sum X_{i} r_{i} d\right)^{3}
$$

where $X_{i}$ is the mole fraction, $U_{i}$ the ultrasonic velocity, and $\rho_{i}$ the density of the mixture. $r_{i}$ is the Rao's specific sound velocity, which is given by $r_{i}=U_{i}^{1 / 3} / \rho_{i}$, and $Z_{i}$ is the acoustic impedance.

\section{Ideal Mixing Relation ( $\left.U_{\mathrm{IMR}}\right)$}

Van Deal and Vangeel [27] suggested the following relation for the velocity of sound:

$$
\frac{1}{\left(X_{1} M_{1}+X_{2} M_{2}\right) * U_{\text {imix }}^{2}}=\frac{X_{1}}{M_{1} U^{2}}+\frac{X_{2}}{M_{2} U^{2}},
$$

where $U_{i \text { mix }}$ is the ideal mixing ultrasonic velocity in liquid mixture, and $U_{1}$ and $U_{2}$ are the velocities of the individual components.

The degree of molecular interaction given as interaction parameter $(\chi)$ by

$$
\chi=\left(\frac{U_{\text {exp }}^{2}}{U_{\text {imix }}^{2}}\right)^{-1}
$$

\section{Results and Discussions}

As applied to basic chemistry, a polar protic solvent has a hydrogen atom attached to a strongly electronegative element (e.g., oxygen) that forms hydrogen bonds, On the other hand; polar aprotic solvents are those solvents whose molecules do not have a hydrogen atom that's attached to an atom of an electronegative element. Polar protic solvent solvate cations and anions effectively while aprotic solvents 


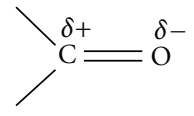

SCHEME 1

do not solvate anions to any appreciable extend. In our study, the alkoxyethanols are polar protic solvents, and try to form hydrogen bond easily as one can expect, and anisaldehyde comes under polar aprotic solvent, since it has a carbonyl group - $\mathrm{CHO}$ in which the hydrogen is attached to the carbon atom which is less electronegative than the oxygen atom. Still bonding in carbonyl group of anisaldehyde is polar, since oxygen is far more electronegative than carbon and so has a strong tendency to pull electrons in a carbon-oxygen bond towards itself. One of the two pairs of electrons that make up a carbon-oxygen double bond is even more easily pulled towards the oxygen, which makes the carbon-oxygen double bond very highly polar (see Scheme 1).

This polarity with a dipole moment of 3.7855 [28] further tries to pull the hydrogen from oxyethanol molecules (protonators), which are already self-associated. This is the key phenomenon occurring in new hydrogen bond formation between heteromolecules. As the carbon chain length increases from methoxyethanol to butoxy ethanol, the tendency to form hydrogen bond decreases owing to stearic hindrance of long carbon chain, and hence, weakening of associations between dissimilar molecules is expected.

The validity of various theoretical approaches of liquids has been tested for the binary systems of Anisaldehyde + methoxyethanol (MOE) + Ethoxyethanol (EOE) + butoxyethanol (BOE) by comparing theoretical sound speeds with those experimentally determined in the temperature range $303-318 \mathrm{~K}$. The experimental values of sound speed for the system along with theoretical values and percentage deviations for Nomoto's Relation $\left(U_{\mathrm{NR}}\right)$, Vandeal Vangael Ideal Mixing Relation $\left(U_{i \text { mix }}\right)$, Impedance Dependence Relation $\left(U_{\mathrm{IR}}\right)$, Rao's specific velocity method $\left(U_{R}\right)$, and Junjie's relation $\left(U_{J}\right)$ are compared for all the three binaries.

It is assumed that all the molecules are spherical in shape, which is not true every time. In Nomoto's theory, it is supposed that the volume does not change on mixing. Therefore, no interaction between the components of liquid mixtures has been taken into account. The assumption for the formation of ideal mixing relation is that the ratio of specific heats of ideal mixtures and the volumes are also equal. Again, no molecular interaction is taken into account. Similarly, as per the assumption for the collision factor theory, the molecules are treated as real nonelastic substances, which is not really the case. But on mixing two liquids, the interaction between the molecules of the two liquids takes place because of presence of various types of forces such as dispersion forces, charge transfer, hydrogen bonding, dipole-dipole and dipole-induced dipole interactions. Thus, the observed deviation of theoretical values of velocity from the experimental values shows that the molecular interaction is taking place between the unlike molecules in the liquid mixture. It can be seen from
Tables 1, 2, and 3 that the theoretical values of ultrasonic velocity computed by various theories show deviations from experimental values. The limitations and approximations incorporated in these theories are responsible for it.

In general, the predictive ability of various ultrasonic theories depends upon the strength of interactions that exist in a binary system. In case strong interactions exist between the molecules of the mixtures, there is much deviation in theoretical prediction of velocity than the molecules of the mixture where less interactions are present.

Data reveal that the sound speed computed from impedance relation, $U_{\text {IR }}$ exhibit more satisfactory agreement with the experimental values in the temperature range $303.15 \mathrm{~K}-318.15 \mathrm{~K}$ than other approaches in the binary systems $\mathrm{AA}+\mathrm{EOE}$ and $\mathrm{AA}+\mathrm{BOE}$, but in case of $\mathrm{AA}+\mathrm{MOE}$, it's by Nomoto $\left(U_{\mathrm{NR}}\right)$.

Table 1 shows that in the system of anisaldehyde + moethxyethanol, there is good agreement between experimental and theoretical values calculated by impedance and Nomoto relations. Here Nomoto relation provides the best result than the result of impedence relation at all the temperatures. However, higher deviations are observed in Rao's specific and slight variations in Junjie's theories.

Anisaldehyde + ethoxyethanol system, as described in Table 2, there is good agreement between experimental and theoretical values in Nomoto relation followed by impedance relations where as higher deviations are observed in van Dael ideal mixing relation, Rao's specific velocity method, and Junjie's relation.

Anisaldehyde and Butoxyethanol showed good agreement between experimental and theoretical values calculated by Rao's specific velocity method and impedance relation. Here Rao's specific velocity method provides the best result than the result of impedance relation at all temperatures. However, higher deviations are observed in Nomoto's relation, van Dael ideal mixing relation and Junjie's theory.

The percentage deviations of the ultrasonic velocity are both negative and positive. Such deviations indicate the nonideal behavior of liquid mixtures. The ratio $U_{\exp }^{2} / U_{\text {imix }}^{2}$ is used as an important tool to measure the nonideality in the mixtures, especially in these cases where the properties other than sound velocity are not known. A perusal of Tables 1, 2, and 3 indicate small deviations from ideality, which may be due to the existence of weak tendency for the formation of association in liquid mixtures.

Looking into the behavior of all three binary mixtures, it can be understood that, positive deviation in velocity are attributed to the molecular associations, complex formations, whereas negative deviations indicate molecular dissociations of an associated species by the addition of solvent.

In our investigation, maximum positive values are observed for $U_{\text {exp }}^{2} / U_{\text {imix }}^{2}$ values for AA+MOE (1.178), than $\mathrm{AA}+\mathrm{EOE}(1.095)$ at nearly equimolar composition at all measured temperatures due to more complex formations between unlike molecules through hydrogen bonding, where as for $\mathrm{AA}+\mathrm{BOE}$ system, it is observed that the values are very less at the same composition (0.988), and the graph showed negative deviation indicating dominance of 


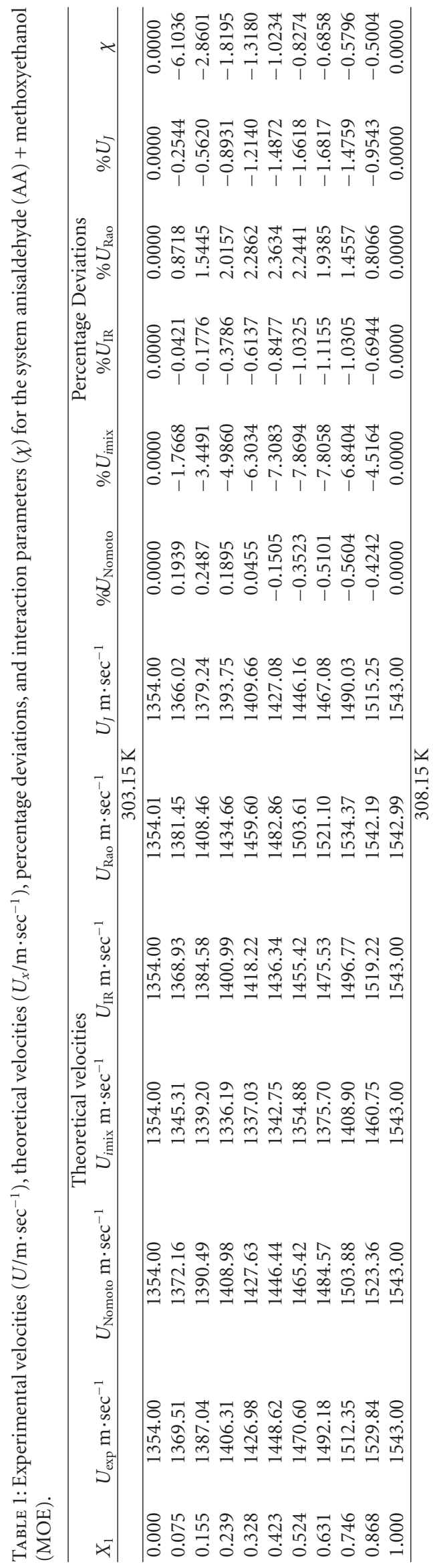

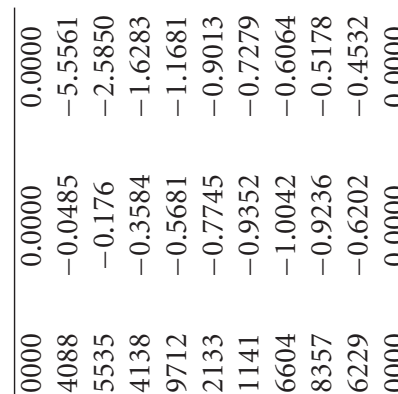

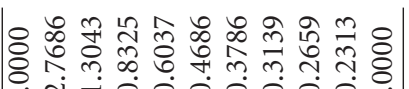

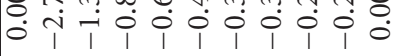

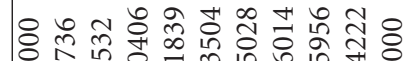

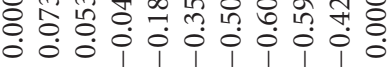

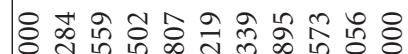

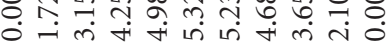

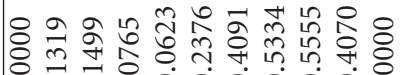

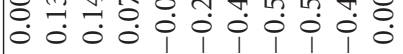

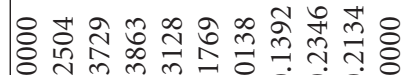

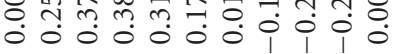

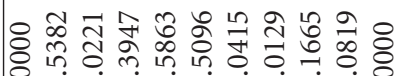

ㅇํㅇำ

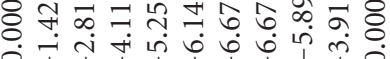
-i $\vec{i}+1$ i

\&

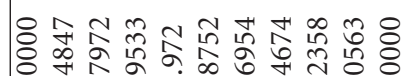

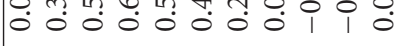

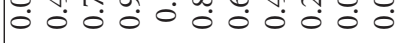

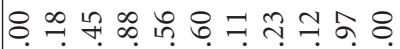

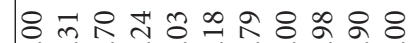

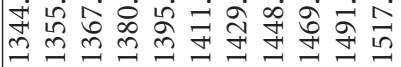
管 4 in

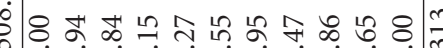

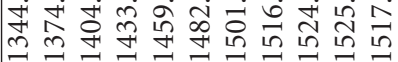

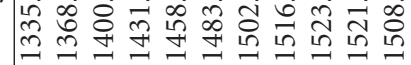

웅

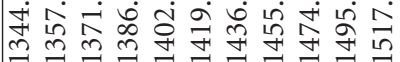

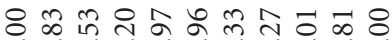

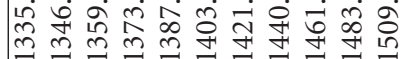

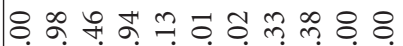
苮

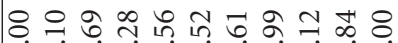
लिं

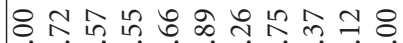

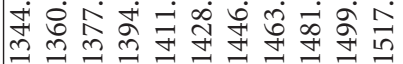

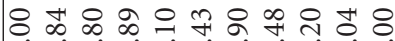

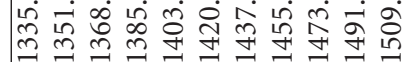

8 ๘

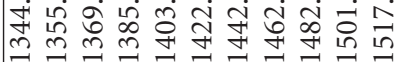

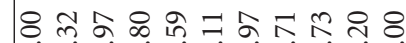

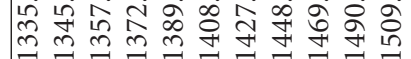

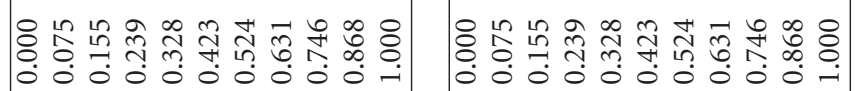




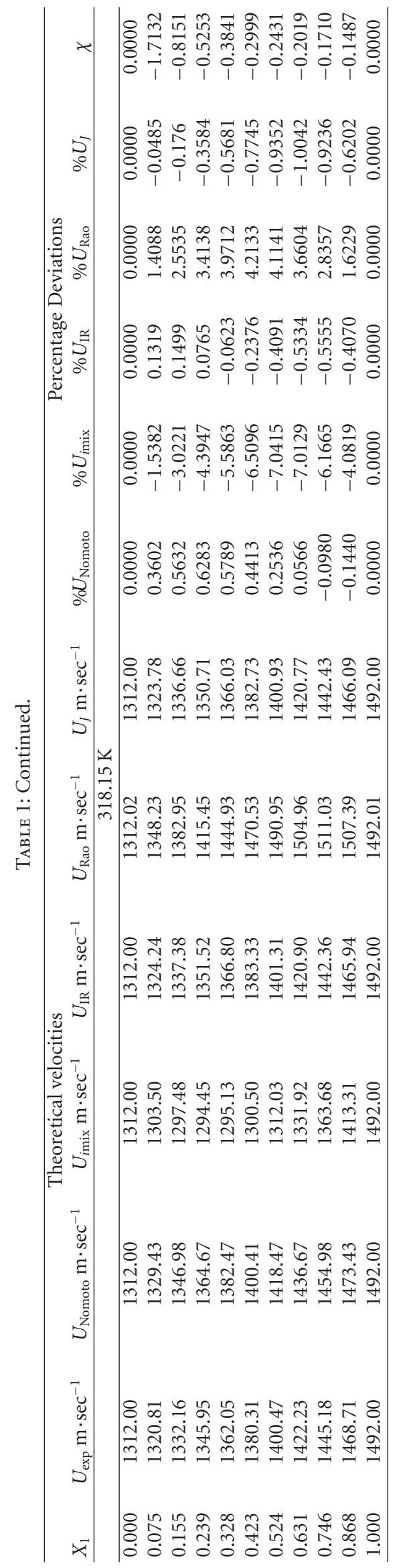




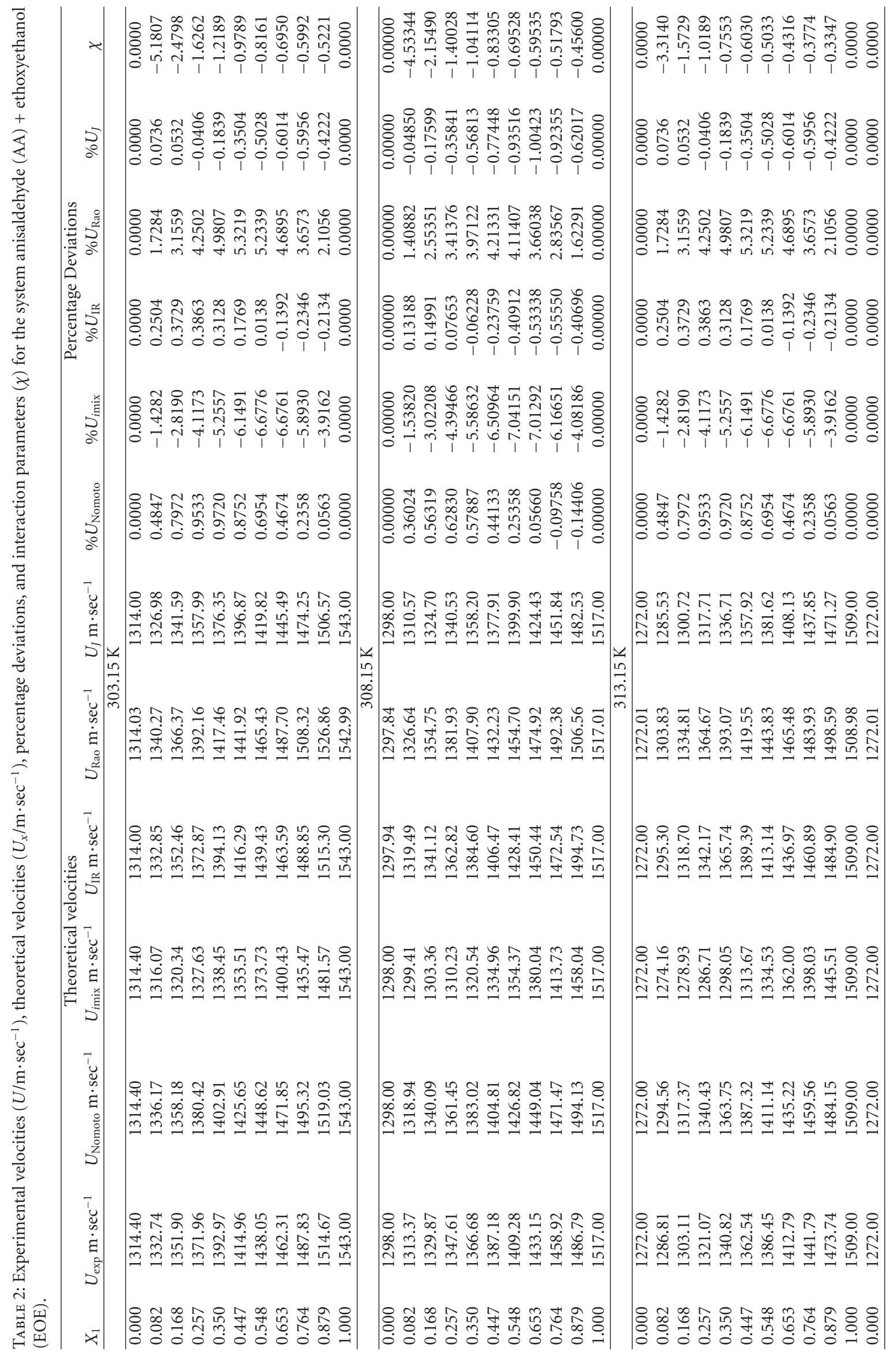




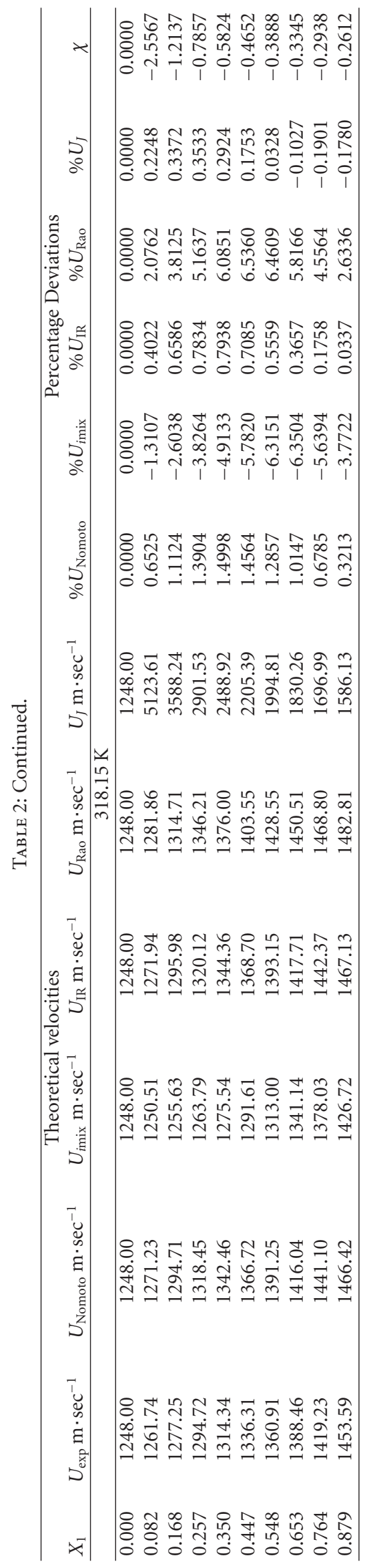




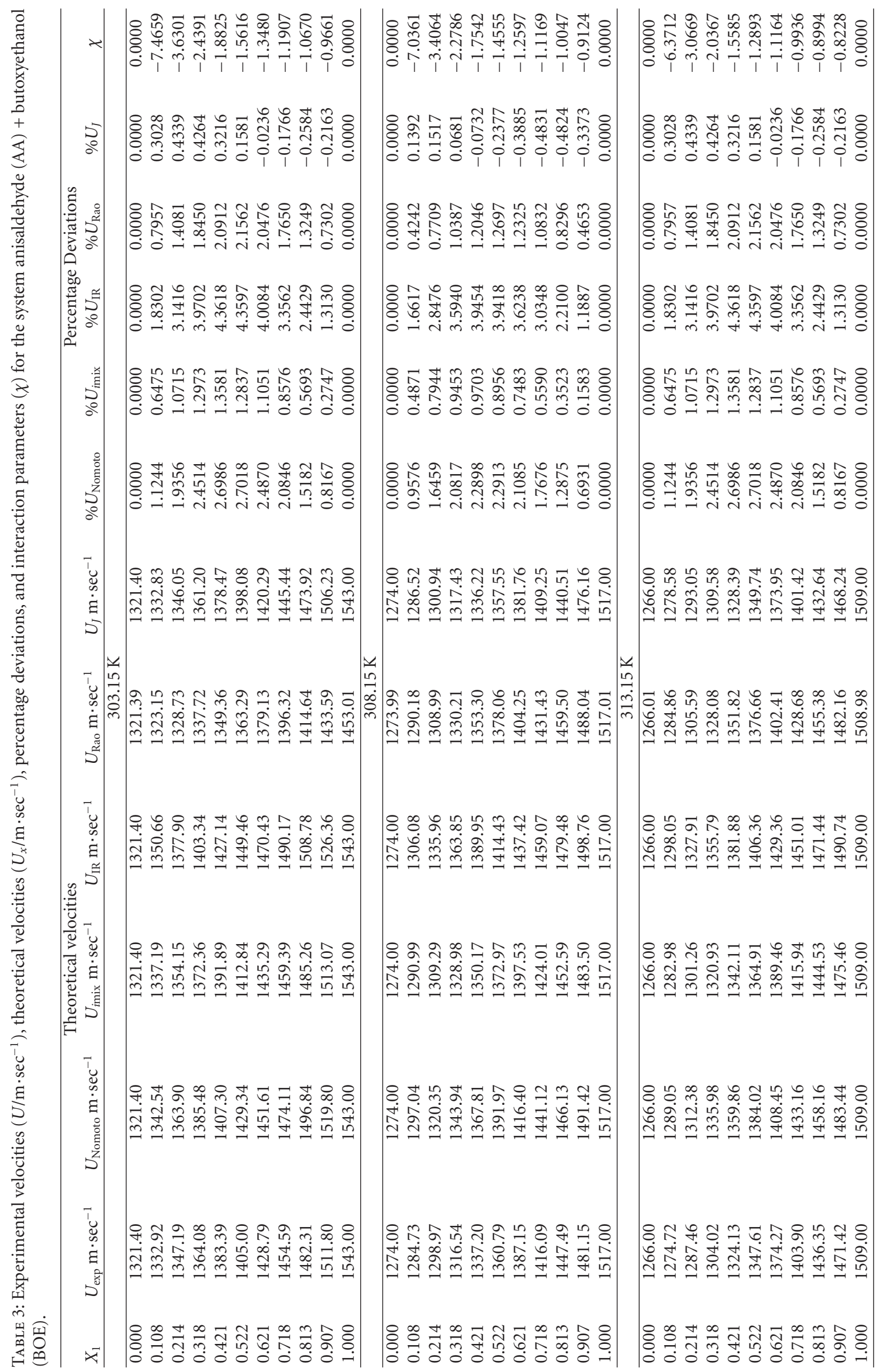




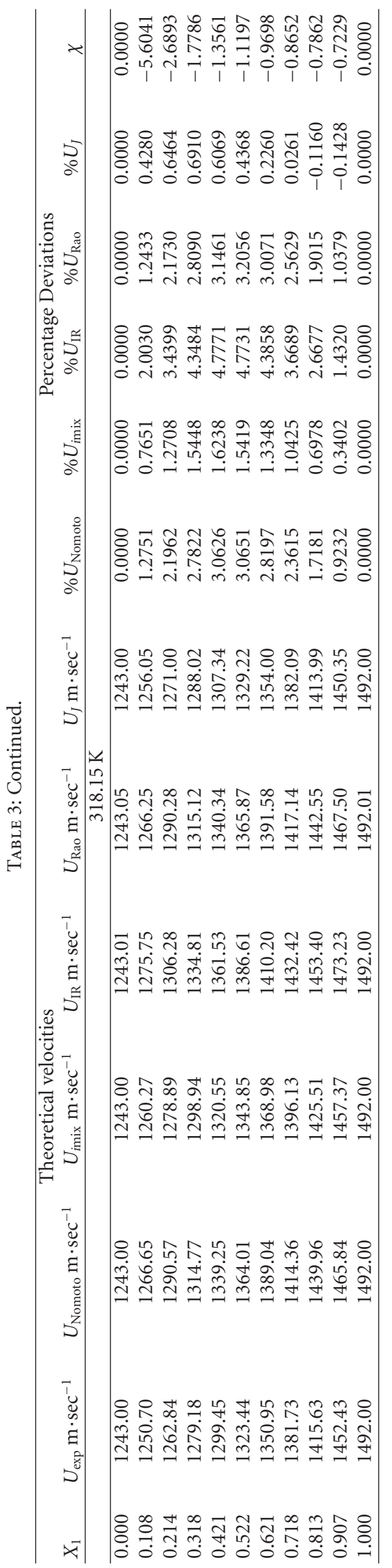


TABLE 4: $U^{2} / U_{\text {imix }}^{2}$ values for AA+MOE, EOE and BOE systems at all four temperatures from $303.15 \mathrm{~K}-318.15 \mathrm{~K}$.

\begin{tabular}{lcccccccccccccccc}
\hline \multicolumn{1}{c}{ AA+MOE } & \multicolumn{1}{c}{ AA+EOE } \\
$X_{1}$ & $303.15 \mathrm{~K}$ & $308.15 \mathrm{~K}$ & $313.15 \mathrm{~K}$ & $318.15 \mathrm{~K}$ & $X_{1}$ & $303.15 \mathrm{k}$ & $308.15 \mathrm{k}$ & $313.15 \mathrm{k}$ & $318.15 \mathrm{k}$ & $X_{1}$ & $303.15 \mathrm{k}$ & $308.15 \mathrm{k}$ & $313.15 \mathrm{k}$ & $318.15 \mathrm{k}$ \\
\hline 0.000 & 1.0000 & 1.0000 & 1.0000 & 1.0000 & 0.0000 & 1.0000 & 1.0000 & 1.0000 & 1.0000 & 0.0000 & 1.0000 & 1.0000 & 1.0000 & 1.0000 \\
0.0753 & 1.0363 & 1.0315 & 1.0292 & 1.0267 & 0.0823 & 1.0255 & 1.0216 & 1.0200 & 1.0180 & 0.1080 & 0.9936 & 0.9903 & 0.9872 & 0.9849 \\
0.1549 & 1.0727 & 1.0633 & 1.0589 & 1.0542 & 0.1679 & 1.0484 & 1.0411 & 1.0382 & 1.0347 & 0.2142 & 0.9898 & 0.9843 & 0.9789 & 0.9751 \\
0.2391 & 1.1077 & 1.0940 & 1.0877 & 1.0812 & 0.2571 & 1.0679 & 1.0579 & 1.0541 & 1.0495 & 0.3184 & 0.988 & 0.9814 & 0.9746 & 0.9698 \\
0.3283 & 1.1391 & 1.1218 & 1.1140 & 1.1060 & 0.3499 & 1.0831 & 1.0711 & 1.0670 & 1.0618 & 0.4209 & 0.9878 & 0.9809 & 0.9734 & 0.9683 \\
0.4231 & 1.1639 & 1.1441 & 1.1353 & 1.1265 & 0.4467 & 1.0929 & 1.0798 & 1.0758 & 1.0704 & 0.5215 & 0.9889 & 0.9823 & 0.9748 & 0.9699 \\
0.5238 & 1.1781 & 1.1572 & 1.1482 & 1.1394 & 0.5477 & 1.0958 & 1.0827 & 1.0793 & 1.0743 & 0.6205 & 0.9910 & 0.9852 & 0.9783 & 0.9738 \\
0.6311 & 1.1765 & 1.1565 & 1.1482 & 1.1402 & 0.6532 & 1.0903 & 1.0784 & 1.0760 & 1.0718 & 0.7178 & 0.9934 & 0.9889 & 0.9831 & 0.9795 \\
0.7457 & 1.1522 & 1.1358 & 1.1292 & 1.1231 & 0.7636 & 1.0743 & 1.0650 & 1.0636 & 1.0607 & 0.8134 & 0.9960 & 0.9930 & 0.9887 & 0.9862 \\
0.8684 & 1.0968 & 1.0869 & 1.0832 & 1.0799 & 0.8790 & 1.0452 & 1.0398 & 1.0394 & 1.0380 & 0.9075 & 0.9983 & 0.9968 & 0.9945 & 0.9932 \\
1.0000 & 1.0000 & 1.0000 & 1.0000 & 1.0000 & 1.0000 & 1.0000 & 1.0000 & 1.0000 & 1.0000 & 1.0000 & 1.0000 & 1.0000 & 1.0000 & 1.0000 \\
\hline
\end{tabular}

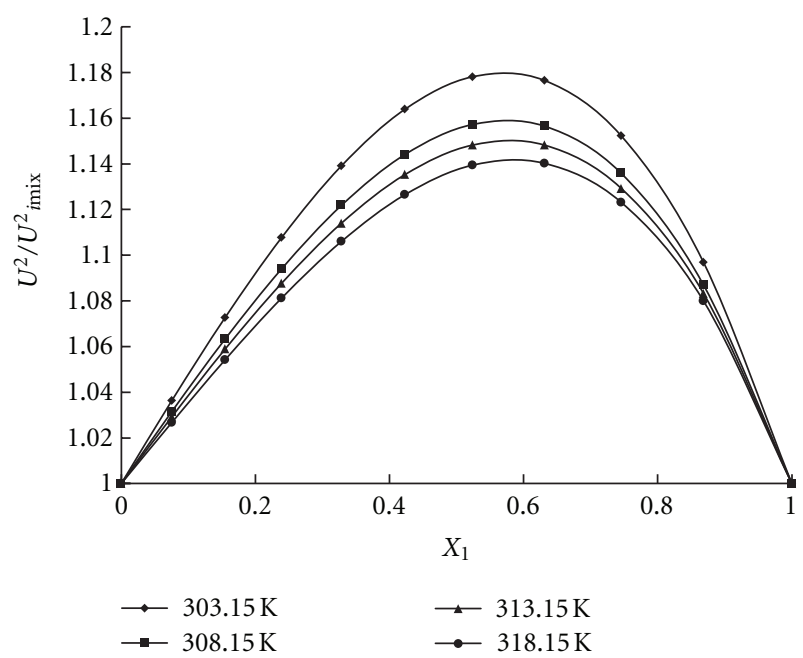

Figure 1: Variation of $\left(U^{2} / U_{\text {imix }}^{2}\right)$ for the system $(\mathrm{AA}+\mathrm{MOE})$ at temperatures $303.15 \mathrm{~K}, 308.15 \mathrm{~K}, 313.15 \mathrm{~K}$, and $318.15 \mathrm{~K}$. Against mole fraction of AA $\left(X_{1}\right)$.

dispersion forces more than associations. Same is observed in our thermodynamic and FTIR analysis, discussed in our separate paper. The $U_{\exp }^{2} / U_{i \text { mix }}^{2}$ values of all three binaries have been shown in Table 4 .

Figures 1,2, and 3 represent the variation of $U_{\text {exp }}^{2} / U_{\text {imix }}^{2}$ with mole fraction of anisaldehyde with all three alkoxyethanols. It is observed that in anisaldehyde + methoxyethanol anisaldehyde + ethoxyethanol systems it is maximum at approximately $0.52 \mathrm{M}$ and in the case of and anisaldehyde and butoxyethanol systems it is minimum at approximately $0.522 \mathrm{M}$ at all the temperatures.

The interaction parameter characterizing a system varies with the compostion, molar mass, and temperature. It is employed to account for the contribution of noncombinatorial entropy of mixing and the enthalpy of mixing to the Gibbs energy of mixing. When the values of interaction parameters show positive sign, it represents strong interaction between the mixing molecules. In systems studied, we observed that the interaction parameters are negative for all

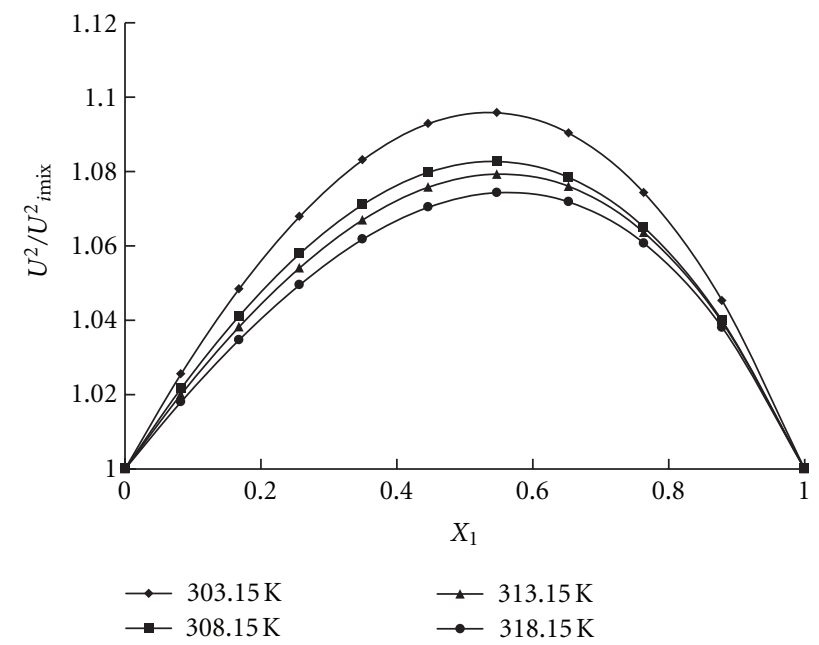

Figure 2: Variation of $\left(U^{2} / U_{\text {imix }}^{2}\right)$ for the system $(\mathrm{AA}+\mathrm{EOE})$ at temperatures $303.15 \mathrm{~K}, 308.15 \mathrm{~K}, 313.15 \mathrm{~K}$ and $318.15 \mathrm{~K}$. Against mole fraction of AA $\left(X_{1}\right)$.

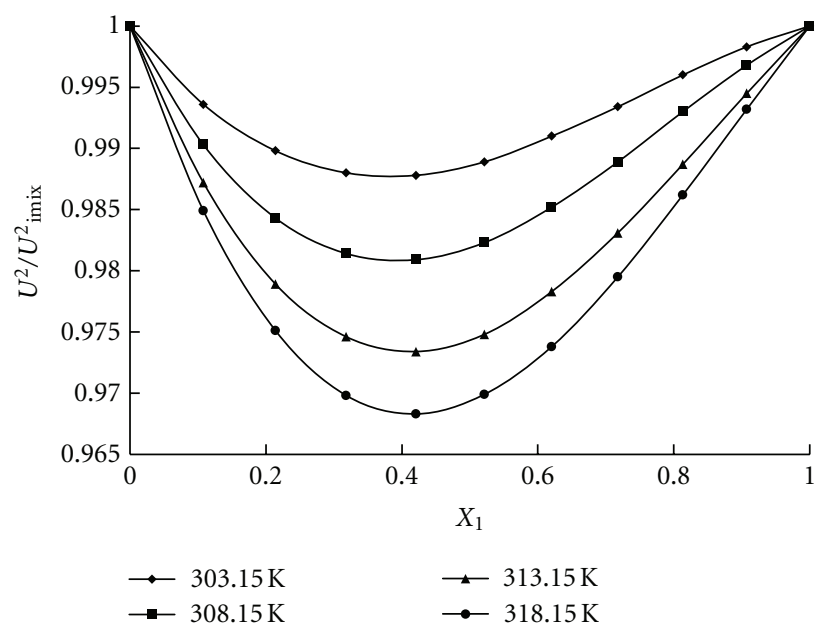

FIgure 3: Variation of $\left(U^{2} / U_{\text {imix }}^{2}\right)$ for the system $(\mathrm{AA}+\mathrm{BOE})$ at temperatures $303.15 \mathrm{~K}, 308.15 \mathrm{~K}, 313.15 \mathrm{~K}$ and $318.15 \mathrm{~K}$. Against mole fraction of AA $\left(X_{1}\right)$. 
the given mixtures, indicating weaker interactions, though hydrogen bonding is present, which decrease from MOE to BOE.

The deviations between theoretical and experimental value of ultrasonic velocities decrease with increase of temperature due to breaking of hetero- and homomolecular clusters at higher temperatures [29]. On increasing the temperature, the ultrasonic velocity values decrease in the three binary liquid mixtures. This is probably due to the fact that the thermal energy activates the molecule, which would increase the rate of association of unlike molecules.

\section{Conclusions}

It may be concluded that out of five theories and relations discussed above the Nomoto's relation, van Deal ideal mixing relation and Impedance relation provided good results. Thus, the linearity of molar sound velocity and additivity of molar volumes, as suggested by Nomoto Van Dael and Vangeel, and Impedance relation, in deriving the empirical relations have been truly observed in the aforementioned binary liquid mixtures. The success of Nomoto's relation in predicting the experimental ultrasonic velocities for non-polar-polar liquid mixtures has also been emphasized by others $[30,31]$.

\section{References}

[1] G. V. Rama Rao, A. Viswanatha Sarma, J. Siva Rama Krishna, and C. Rambabu, "Theoretical evaluation of ultrasonic velocities in binary liquid mixtures of o-chlorophenol at different temperatures," Indian Journal of Pure and Applied Physics, vol. 43, no. 5, pp. 345-354, 2005.

[2] P. Vasantharani, S. Muthu Shailaja, A. N. Kannappan, and R. Ezhil Pavai, "Theoretical evaluation of ultrasonic velocity in organic liquid mixtures," Journal of Applied Sciences, vol. 8, no. 12, pp. 2329-2332, 2008.

[3] J. D. Pandey, A. K. Singh, and R. Dey, "Novel approach for prediction of ultrasonic velocity in quaternary liquid mixtures," Pramana, vol. 64, no. 1, pp. 135-139, 2005.

[4] J. D. Pandey, R. Dey, and D. K. Dwivedi, "Ultrasonic velocity of binary systems at elevated pressures," Pramana, vol. 52, no. 2, pp. 187-193, 1999.

[5] T. Sumathi and J. U. Maheswari, "Ultrasonic and theoretical studies of some ternary liquid mixtures at Various temperatures," Indian Journal of Pure and Applied Physics, vol. 47, no. 11, pp. 782-786, 2009

[6] S. Baluja and S. Oza, "Studies of some acoustical properties in binary solutions," The Journal of Pure and Applied Ultrasonics, vol. 24, p. 580, 2002.

[7] A. Ali, A. Yasmin, and A. K. Nain, "Study of intermolecular interactions in binary liquid mixtures through ultrasonic speed measurement," Indian Journal of Pure and Applied Physics, vol. 40, no. 5, pp. 315-322, 2002.

[8] M. Rastogi, A. Awasthi, M. Gupta, and J. P. Shukla, "Ultrasonic investigations of X...HO bond complexes," Indian Journal of Pure and Applied Physics, vol. 40, no. 4, pp. 256-263, 2002.

[9] A. Ali, A. K. Nain, and S. Hyder, "Ultrasonic study of molecular interaction in binary liquid mixtures at 308.15," Journal of Pure and Applied Ultrasonics, vol. 23, p. 73, 2001.

[10] O. Nomoto, Journal of the Physical Society of Japan, vol. 4, p. $280,1949$.
[11] O. Nomoto, "Empirical Formula for Sound Velocity in Liquid Mixtures," Journal of the Physical Society of Japan, vol. 13, pp. 1528-1532, 1958.

[12] W. van Dael and E . Vangeel, in Proceedings of the International Conference on Calorimetry and Thermodynamics, p. 555, Warsaw, Poland, 1955.

[13] S. Baluja and R. H. Parsania, "Studies on acoustical properties of diclofanac sodium methanol-water system at $30^{\circ} \mathrm{C}$," Asian Journal of Chemistry, vol. 9, no. 1, pp. 149-152, 1997.

[14] K. Rayapa Reddy, D. Bala Karuna Kumar, C. Rambabu, and G. Srinivasa Rao, "Theoretical evaluation of ultrasonic velocities in binary liquid mixtures of $N$-Methyl-2-pyrrolidone at different temperatures with Some cyclic compounds," E-Journal of Chemistry, vol. 9, no. 2, pp. 553-562, 2012.

[15] Z. Junjie, Journal of University of Science and Technology of China, vol. 14, p. 298, 1984.

[16] R. T. Langeman and J .E. Correy, "Velocity of sound as a bond property," Journal of Chemical Physics, vol. 10, no. 12, p. 759, 1942.

[17] G. R. Rendall, "Dispersion of sound velocity in some alcohols," Proceedings of the Indian Academy of Sciences A, vol. 16, no. 6, pp. 369-378, 1942.

[18] D. Auslander and L. Onitni, Acustica, vol. 24, p. 205, 1971.

[19] K. Samal and S. C. Misra, "Ultrasonic absorption in binary mixtures of carbon disulphide in comparison with Bauer's theory," Journal of the Physical Society of Japan, vol. 32, no. 6, pp. 1615-1618, 1972.

[20] R. A. Aziz and D. H. Bowman, "The law of corresponding states as applied to sound velocity in liquids consisting of elliptical molecules," Canadian Journal of Physics, vol. 50, no. 7, pp. 721-727, 1972.

[21] G. R. Poole, R. A. Aziz, and C. C. Lim, "An examination of the relationship between sound velocity and density in liquids," Canadian Journal of Physics, vol. 50, pp. 646-654, 1972.

[22] B. A. Younglove, "Speed of sound in fluid parahydrogen," Journal of the Acoustical Society of America, vol. 38, pp. 433438, 1965 .

[23] A. Prash, S. Prakash, and Q. Prakash, Proceedings of the National Academy of Sciences, vol. 55, p. 114, 1985.

[24] R. Sabeson, Natarajan, and R. Varadha Rajan, Indian Journal of Pure and Applied Physics, vol. 25, p. 489, 1987.

[25] A. Pal and Y. P. Singh, "Excess molar volumes and apparent molar volumes of some amide + water systems at 303.15 and 308.15 K," Journal of Chemical \& Engineering Data, vol. 40, no. 4, pp. 818-822, 1995.

[26] J. A. Reddick, W. B. Bungar, and T . Sakano, Organic Solvents: Physical Properties and Methods of Purification, WileyInterscience, New York, NY, USA, 4th edition, 1986.

[27] M. J. Blandamer and D. Waddington, "Analysis of sound velocities in aqueous mixtures in terms of excess isentropic compressibilities," Journal of Physical Chemistry, vol. 74, no. 12, pp. 2569-2570, 1970.

[28] S. Gunasekaran, S. Seshadri, S. Muthu, S. Kumaresan, and R. Arunbalaji, "Vibrational spectroscopy investigation using ab initio and density functional theory on p-anisaldehyde," Spectrochimica Acta A, vol. 70, no. 3, pp. 550-556, 2008.

[29] P. S. Nikam, B. S. Jagdale, A. B. Sawant, and M. Hasan, Journal of Pureand Applied Ultrasonics, vol. 22, p. 115, 2000.

[30] A. Ali and A. K. Nain, "Study of molecular interactions in non-aqueous binary liquid mixtures through ultrasonic measurements," Journal of Pure and Applied Ultrasonics, vol. 22, p. 10, 2000. 
[31] A. Ali, A. K. Nain, V. K. Sharma, and S. Ahmad, "Molecular interactions in binary mixtures of tetrahydrofuran with alkanols (C6,C8,C10): an ultrasonic and volumetric study," Indian Journal of Pure and Applied Physics, vol. 42, no. 9, pp. 666-673, 2004. 


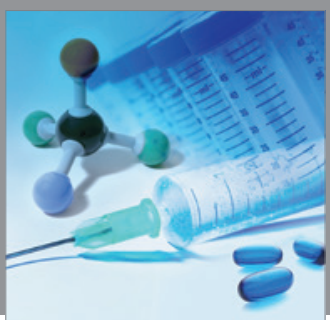

International Journal of

Medicinal Chemistry

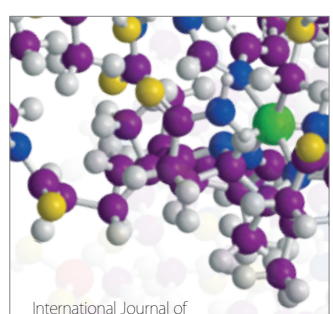

Carbohydrate Chemistry

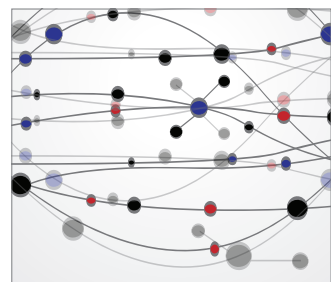

The Scientific World Journal
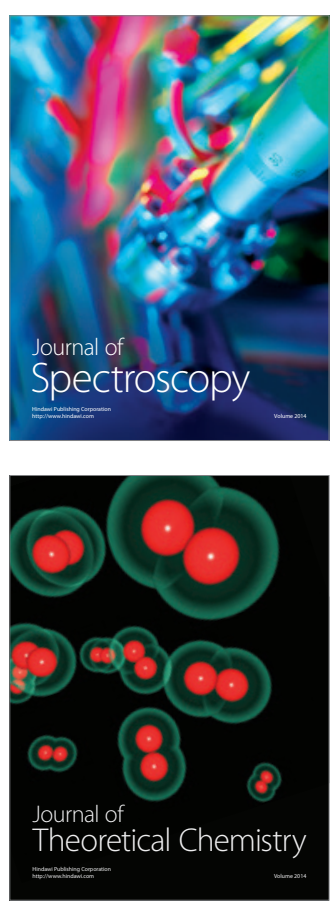
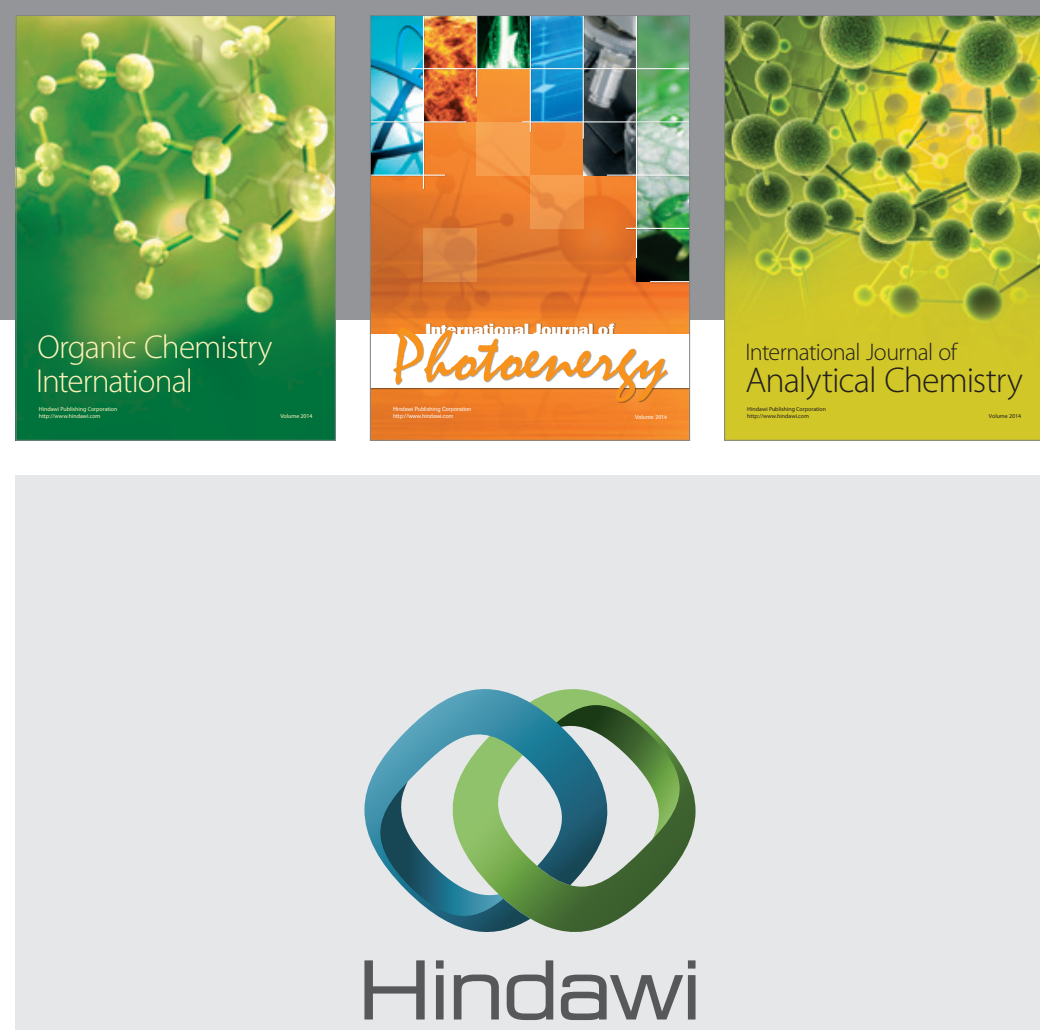

Submit your manuscripts at

http://www.hindawi.com
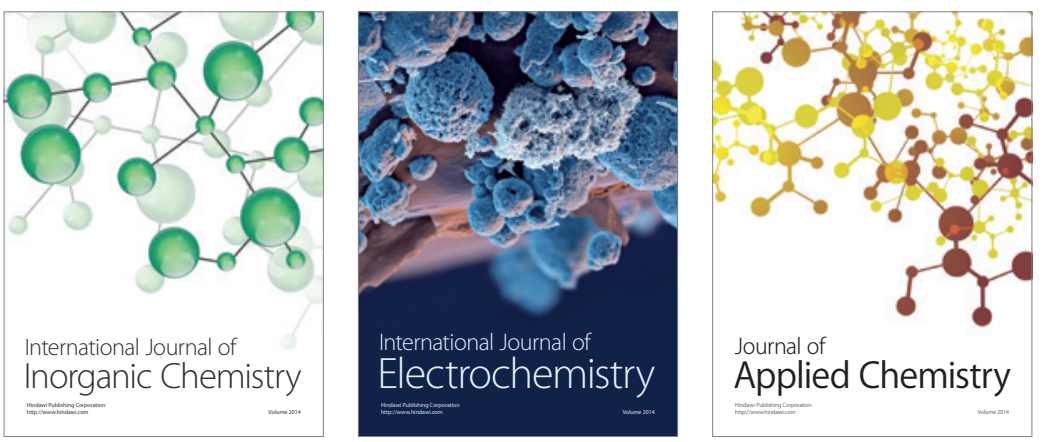

Journal of

Applied Chemistry
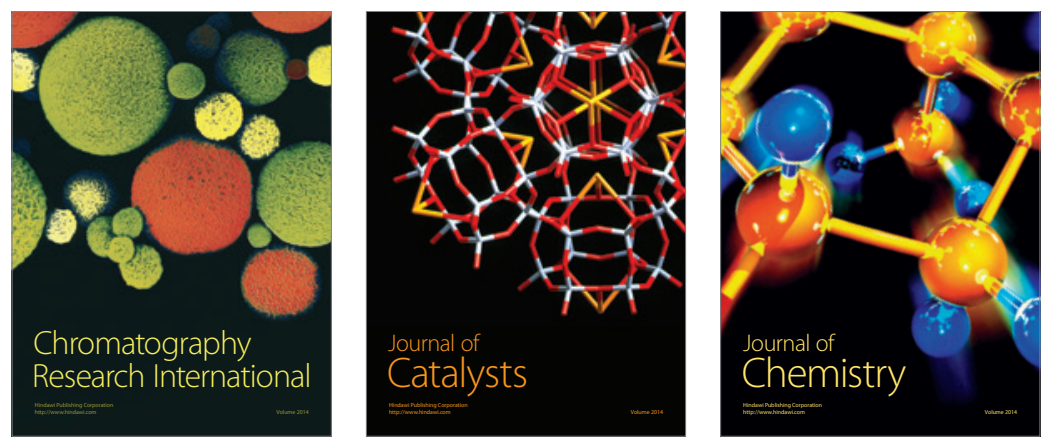
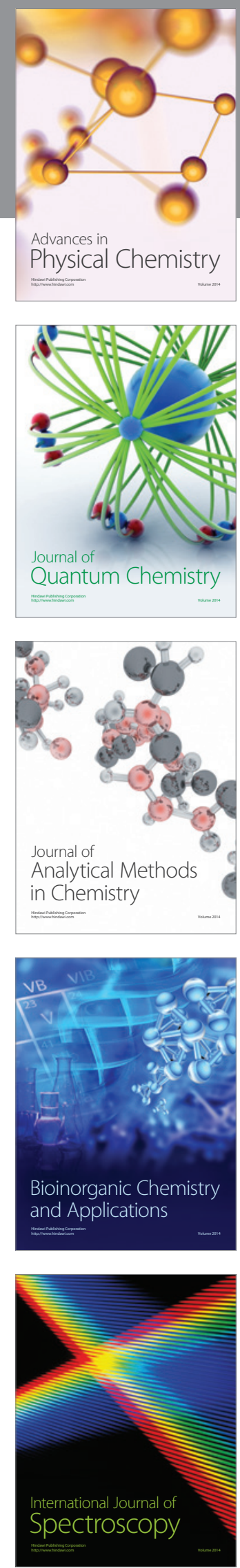